\title{
Severe Acute Respiratory Syndrome Coronavirus 2 (SARS-CoV-2) Infection During Pregnancy In China: A Retrospective Cohort Study
}

Ming-Zhu Yin, MD ${ }^{1,2,79^{*}}$, Li-juan Zhang, $\mathrm{MD}^{3,8^{*}}$, Guang-Tong Deng, $\mathrm{MD}^{2,7 *}$, Chao-Fei Han, $\mathrm{MD}^{4,8^{*}}$, Min-Xue Shen, $\mathrm{phD}^{2,7^{*}}$, Hong-Yin Sun, $\mathrm{MD}^{2,7}$, Fu-Rong Zeng, $\mathrm{MD}^{2,7}$, Wei Zhang, MD ${ }^{3,7}$, Lan Chen, $\mathrm{MD}^{5,8}$, Qing-Qing Luo, MD ${ }^{5,8}$, Du-Juan Yao, $\mathrm{MD}^{5,8}$, Min Wu, MD ${ }^{5,8}$, Shi-Huan Yu, MD ${ }^{3,8^{*}}$, Hui Chen, $\mathrm{MD}^{5,8^{*}}$, David Baud, $\mathrm{MD}^{6,9 *}$, Xiang Chen, $\mathrm{MD}^{2,7 *}$

${ }^{1}$ Hunan Engineering Research Center of Obstetrics and Gynecological Disease

${ }^{2}$ Department of Dermatology, Hunan Engineering Research Center of Skin Heath and Disease, Xiangya Hospital, Central South University, Changsha, Hunan, China;

${ }^{3}$ Department of Respiratory and Critical Care Medicine, The First Affiliated Hospital of Harbin Medical University, Harbin, China;

${ }^{4}$ Department of Burn and Plastic Surgery, The Third Xiangya Hospital of Central South University, Changsha, Hunan, China.

${ }^{5}$ Department of Obstetrics and Gynecology, Union Hospital, Tongji Medical College, Huazhong University of Science and Technology, Wuhan, Hubei, China;

${ }^{6}$ Materno-fetal and Obstetrics Research Unit, Department "Femme-Mère-Enfant”, University Hospital, Lausanne, Switzerland.

${ }^{7}$ CVID-19 Research Group in Hunan

${ }^{8}$ Support Wuhan Medical Team

${ }^{9}$ COVI-Preg group

* Contributed equally to this article.

Corresponding to Ming-Zhu Yin, the Hunan Engineering Research Center of Obstetrical and Gynecological Disease \& Hunan Engineering Research Center of Skin Heath and Disease, Department of Dermatology, Xiangya Hospital, Central South University, No. 87 Xiangya Rd, Changsha 410008, China, or at yinmingzhu2008@126.com, Tel: +86-15873141982

or

Shi-Huan Yu yushihuan2000@126.com

Hui Chen chinachen67@hust.edu.cn

David Baud David.Baud@chuv.ch

Xiang Chen chenxiangck@126.com 


\section{Summary}

Background Severe acute respiratory syndrome coronavirus 2 (SARS-CoV-2) has been identified as the cause of the ongoing worldwide epidemic of Coronavirus Disease 2019 (COVID-19) in China and worldwide. However, there were few studies about the effects of SARS-CoV-2 infection on pregnant women.

Methods In this retrospective cohort study, we enrolled 31 pregnant women and 35 non-pregnant women from Jan 28 to Feb 28, 2020 to evaluate the effects of SARS-CoV-2 infection during pregnancy. Inflammatory indices were used to assess the severity of COVID-19. Evidence of vertical transmission was determined by laboratory confirmation of SARS-CoV-2 in amniotic fluid, placenta, neonatal throat and anal swab and breastmilk samples.

Findings Compared with non-pregnant women, pregnant women had a significantly lower proportion of fever $(54.8 \%$ vs. $87.5 \%, p=0.006)$, a shorter average interval from onset to hospitalization $(7 \cdot 80$ $\pm 7 \cdot 0 \mathrm{~d}$ vs. $13 \cdot 2 \pm 8 \cdot 2 \mathrm{~d}, p=0.005)$, and a higher proportion of severe or critical COVID-19 (32.3\% vs. $11.4 \%, p=0.039)$. Neutrophil-to-lymphocyte ratio (NLR) and systematic immune-inflammation-based prognostic index (SII) were significantly higher on admission in severe/critical pneumonia group than moderate pneumonia group. We could not detect the presence of SARS-CoV-2 by RT-PCR in amniotic fluid, placenta, neonatal throat and anal swab and breastmilk samples.

Interpretation The clinical symptoms of COVID-19 in pregnant women were insidious and atypical, compared with those in non-pregnant patients. SII and NLR could be a useful marker to evaluate the severity of COVID-19. There was no evidence of vertical transmission during pregnancy with SARS-CoV-2 infection.

Funding National Natural Science Foundation of China and Research Funds for the Central Universities. 


\section{Research in context \\ Evidence before this study}

We searched PubMed, Embase and Web of science for articles published up to March 1st, 2020, using the keywords ("novel coronavirus" OR "2019 novel coronavirus" OR "2019-nCoV” OR COVID-19 OR SARS-CoV-2) AND (pregnancy OR "maternal infection" OR "fetal infection") AND "Cohort studies".

We identified no published cohort studies on pregnant women with the 2019 novel coronavirus disease (COVID-19) infection.

\section{Added value of this study}

For this retrospective cohort study, we reviewed clinical records, laboratory findings, and chest CT scans from 31 pregnant women and 35 non-pregnant women from Jan 28 to Feb 28, 2020 to evaluate the effects of SARS-CoV-2 infection during pregnancy. Inflammatory indices were used to assess the severity of COVID-19. Evidence of vertical transmission was determined by laboratory confirmation of SARS-CoV-2 in amniotic fluid, placenta, neonatal throat and anal swab and breastmilk samples. Compared with non-pregnant women, pregnant women had a significantly lower proportion of fever ( $54.8 \%$ vs. $87.5 \%, \mathrm{p}=0.006)$, a shorter average interval from onset to hospitalization $(7.80 \pm 7 \cdot 0 \mathrm{~d}$ vs. $13 \cdot 2 \pm 8 \cdot 2 \mathrm{~d}, \mathrm{p}=0.005)$, and a higher proportion of severe or critical COVID-19 (32.3\% vs. $11.4 \%$, $\mathrm{p}=0.039$ ). Neutrophil-to-lymphocyte ratio (NLR) and systematic immune-inflammation-based prognostic index (SII) were significantly higher on admission in severe/critical pneumonia group than moderate pneumonia group. Amniotic fluid, placenta, neonatal throat and anal swab and breastmilk samples were tested for SARS-CoV-2 by RT-PCR and all results were negative.

\section{Implications of all the available evidence}

The clinical symptoms of COVID-19 in pregnant women were insidious and atypical, compared with those in non-pregnant patients. SII and NLR could be a useful marker to evaluate the severity of COVID-19. There was no evidence of vertical transmission during pregnancy with SARS-CoV-2 infection. 


\section{Introduction}

Severe acute respiratory syndrome coronavirus 2 (SARS-CoV-2) has been identified as the cause of the ongoing worldwide epidemic of Coronavirus Disease 2019 (COVID-19) first in China and then worldwide. $^{1-5}$ As of March 9, 2020, a total of 113, 702 cases had been reported, including 4, 012 deaths globally. ${ }^{6}$ Phylogenetic analysis revealed that SARS-CoV-2 was a novel single-stranded ribonucleic acid (RNA) betacoronavirus which resembled severe acute syndrome coronavirus (SARS-CoV) ${ }^{5}$. However, SARS-CoV-2 is far more severe and infectious than SARS-CoV, and has become a global health emergency. ${ }^{4,7-9}$

Although numerous studies have illuminated the clinical characteristics and outcomes of general population with COVID-19, ${ }^{2,8}$ little has been reported about the effects of SARS-CoV-2 infection on pregnant women. ${ }^{10,11}$ To our knowledge, a study by Huang and colleagues reported that the clinical characteristics of nine pregnant women with COVID-19 resembled those in the general population. ${ }^{12}$ Another study including ten pregnant patients demonstrated that perinatal SARS-CoV-2 infection had adverse effects on newborns such as fetal distress and even death. ${ }^{13}$ Moreover, a case described that a pregnant woman with COVID-19 delivered a healthy neonate with no evidence of SARS-CoV-2 infection during her 30 weeks pregnancy. ${ }^{14}$ However, these series of cases had limitations for their small size and lack of control group. Therefore, there are still debates on whether pregnant women have a different disease course and outcomes, considering the physiological changes in cell-mediated immunity and pulmonary function during pregnancy. ${ }^{15}$

In this study, we conducted retrospective cohort study to compare the effects of SARS-CoV-2 infection on pregnant women and non-pregnant women. We hope these findings will facilitate efforts, both in China and globally, to make and manage public health planning for pregnant women with COVID-19.

\section{Materials and methods}

\section{Study design and participants}

For this retrospective cohort study, we included two cohorts of female inpatients (20-40 years old, female) from Jan 28 to Feb 28, 2020, at Wuhan Union and Tongji hospitals of Huazhong University of Science and Technology. All female patients were diagnosed as COVID-19 based on the New Coronavirus Pneumonia Prevention and Control Program (6th edition) published by the National Health Commission of China. ${ }^{16}$ A total of 68 patients were initially enrolled, and 2 patients were excluded from our study because they had tumors with COVID-19 (one had cervical cancer and the other had lymphoma in non-pregnant group). Finally, we recruited 66 hospitalized patients with COVID-19 including 31 pregnant women and 35 non-pregnant women. All the patients were PCR-confirmed cases in our study. The clinical outcomes were monitored up to March 8, 2020, the last day of follow-up. Ethical approval for the study was obtained from the Ethics Committee of Wuhan Union and Tongji hospitals of Huazhong University of Science and Technology, and written informed consent was waived by the Ethics Commission of the designated hospital for the emergency of COVID-19.

\section{Data collection}

We obtained demographic, clinical, laboratory, and radiological data from electronic medical records 
with data collection form. Two study investigators $(\mathrm{CH}, \mathrm{LZ})$ independently collected and validated the data. Maternal throat swab samples from the upper respiratory tract were collected at admission in viral-transport medium. Amniotic fluid samples, placenta samples, neonatal throat and anal swab samples were collected immediately after delivery in the operating room. Breastmilk samples were also collected for SARS-CoV-2 testing to evaluate the evidence of vertical transmission. The presence of SARS-CoV-2 was detected with the Chinese Center for Disease Control and Prevention (CDC) recommended kit (BioGerm, Shanghai, China), following WHO guidelines for qRT-PCR. ${ }^{17}$ The primers used have been described previously. ${ }^{12}$

\section{Definitions}

According to the New Coronavirus Pneumonia Prevention and Control program (6th edition), ${ }^{16}$ COVID-19 is classified into four types including mild, moderate, severe, and critical pneumonia. Mild pneumonia means asymptomatic infection or mild clinical symptoms without abnormal chest imaging findings. Moderate pneumonia is defined as having both clinical symptoms and abnormal chest imaging findings. Patients are diagnosed as severe pneumonia when disease progresses to meet any of the following conditions: (1) significantly increased respiration rate: $R R \square \geq \square 30 / \mathrm{min}$; (2) oxygen saturation $\square \leq \square 93 \%$ in the rest state; (3) $\mathrm{PaO} 2 / \mathrm{FiO} 2 \leq 300 \mathrm{mmHg}(1 \mathrm{mmHg}=0.133 \mathrm{kPa})$. Critical pneumonia occurs when disease progresses rapidly with any of the following conditions: (1) respiratory failure which requires mechanical ventilation; (2) shock; (3) other organ failures in need of intensive care unit (ICU) monitoring and treatment. Fever was defined when the axillary temperature was equal to or above $37.5^{\circ} \mathrm{C}$. Hospital stay was described as the length of stay of patients at the hospital. Neutrophil-to-lymphocyte ratio (NLR) was measured by the absolute neutrophils count to lymphocytes count. Lymphocyte-to-monocyte ratio (LMR) was measured by absolute lymphocytes count to monocytes count. Platelet-to-lymphocyte ratio (PLR) was calculated by the platelets count to lymphocytes count. Systematic immune-inflammation-based prognostic index (SII) was calculated by platelets/lymphocytes $\times$ neutrophils. Aspartate aminotransferase-to-platelet ratio index (APRI) was calculated by aspartate aminotransferase $($ AST $)$ /upper limit of normal range/platelets $\left(10^{9} / \mathrm{L}\right) \times 100$ (the upper limit of normal for AST was $40 \mathrm{U} / \mathrm{L}$ ). Aminotransferase-to-neutrophil ratio index (ANRI) was measured by AST/absolute neutrophils count.

\section{Statistical analysis}

Continuous variables with normal distribution were expressed as mean \pm standard deviation (SD) and compared with analysis of variance (ANOVA). Continuous data with skewed distribution were presented as median (interquartile range, IQR) and compared with Wilcoxon rank sum test. Categorical variables were summarized as counts (percentages) and compared using the chi-square test or Fisher's exact test. Missing data was not imputed, and was indicated in tables. Subgroup analysis was conducted by gestational period. All the analyses were performed with SPSS software, version 23. P value less than 0.05 was considered statistically significant.

\section{Role of funding source}

This study was supported by grants from the General Program the National Natural Science Foundation of China (81874138) and Research Funds for the Central Universities (2020kfyXGYJ008). 


\section{Results}

\section{Clinical characteristics in pregnant and non-pregnant women with COVID-19}

A total of 66 women of childbearing age (aged 20-40) with COVID-19 were included in our study. The mean age was $31 \cdot 0 \pm 4.3$ years in the pregnant group $(\mathrm{n}=31)$ and $32.9 \pm 4.8$ in the non-pregnant group $(\mathrm{n}$ =35). Among them, $14(21 \cdot 2 \%)$ patients were aged 35-40 years (advanced maternal age). Five (14.3\%) non-pregnant patients were previously infected with hepatitis B virus, and 3 out of 31 pregnant patients reported a medical history of gestational hypertension, diabetes and cardiovascular disease, separately (Table S1). There was no history of other diseases in these patients.

The clinical symptoms of the two groups were compared in Table 1. Fever was the most common symptom in both groups, but pregnant patients had a significantly lower proportion of fever than non-pregnant patients $(54.8 \%$ vs. $87.5 \%, p=0.006)$. Moreover, pregnant patients had a shorter average interval from onset to hospitalization than non-pregnant patients $(7 \cdot 80 \pm 7 \cdot 0 \mathrm{~d}$ vs. $13 \cdot 2 \pm 8 \cdot 2 \mathrm{~d}, p=0 \cdot 005)$, while a higher proportion of pregnant patients was diagnosed as severe or critical COVID-19 than non-pregnant patients $(32.3 \%$ vs. $11.4 \%, p=0.039)$. At present, $52(78.8 \%)$ patients were discharged out of hospital, including $22(71 \%)$ pregnant patients and $30(85.7 \%)$ non-pregnant patients. Mean hospital stay, heart rate, and respiratory rate were not statistically different between these two groups. Mean arterial pressure was lower in pregnant patients than non-pregnant patients $(87 \cdot 2 \pm 10.4$ vs. $96 \cdot 7 \pm 7 \cdot 6$ $\mathrm{mmHg}, p<0.001)$.

Laboratory data demonstrated an increase of white blood cell (WBC) and neutrophil counts in pregnant than non-pregnant patients on admission (WBC count $\left[10^{9} / \mathrm{L}\right], 6 \cdot 95 \pm 2.98$ vs. $5 \cdot 11 \pm 2 \cdot 13, p=0 \cdot 005$; neutrophil count $\left[10^{9} / \mathrm{L}\right], 5 \cdot 82 \pm 2.59$ vs. $3.21 \pm 1.89, p<0.001$ ) (Table 2 ). Prothrombin time and activated partial thromboplastin time on admission were shorter in pregnant than non-pregnant patients (prothrombin time [s], $11.9 \pm 1.5$ vs. $13.6 \pm 0.7, p<0.001$; activated partial thromboplastin time [s], $36 \cdot 1 \pm 4 \cdot 1$ vs. $39 \cdot 6 \pm 5 \cdot 1, p=0 \cdot 005$ ), while D-dimer level were higher in pregnant than non-pregnant patients $(0 \cdot 91, \mathrm{IQR}[0 \cdot 58-1 \cdot 32]$ vs. $0 \cdot 36$, IQR [0·26-0.71], $p=0 \cdot 003)$. In addition, pregnant patients were more likely to have a higher level of procalcitonin on admission than non-pregnant patients (30 [96.8\%] vs. 21 [65.6\%], $p=0.002)$. Level of aspartate aminotransferase were increased in nine (13\%) patients, including seven $(22.6 \%)$ pregnant and two (5.7\%) non-pregnant patients (Table 2). Though the concentrations of alanine transaminase and serum creatinine were statistically significant, there was no difference in the proportion of abnormal levels of alanine transaminase and serum creatinine between these two groups. All the enrolled patients underwent the chest CT scan (Fig. S1). Sixty-four (97\%) patients showed patchy shadows or ground glass, consisting of $30(96 \cdot 8 \%)$ pregnant and $34(97 \cdot 1 \%)$ non-pregnant patients. In addition, pregnant and non-pregnant women were administered antiviral therapy (32.2\% vs. $57 \cdot 1 \%)$, glucocorticoid therapy (35.4\% vs. $14.3 \%)$ and oxygen therapy (64.5\% vs. $42 \cdot 8 \%)$.

\section{Inflammatory indices between moderate and severe/critical of pregnant and non-pregnant patients with COVID-19}

Considering that inflammatory cytokine storm was the main lethal factor of infectious pneumonia such as SARS and Middle East Respiratory Syndrome (MERS), ${ }^{18-21}$ we compared the levels of interleukin (IL)-6 and some inflammatory indices including NLR, LMR, PLR, SII, ANRI and APRI, in pregnant and non-pregnant patients (Table 2). Higher levels of IL-6 (3·7, IQR [2·6-5·6] vs. 1·1, IQR [1·1-3·1], $p$ < 0.001), NLR (4.21, IQR [2.88-5.61] vs. 2.31, IQR [1.33-2.54], $p<0.001)$, SII (841.1, IQR [53.2-1175.4] vs. 464.9, IQR [235.7-761·0], $p<0 \cdot 001)$ and APRI (0.33, IQR [0.24-0.42] vs. 0.21, 
IQR [0.16-0.29], $p=0 \cdot 001)$ were found in pregnant patients compared with non-pregnant patients. Evaluated IL- 6 has been reported to be positively associated with the severity of COVID-19. ${ }^{22,23}$ We reasoned that increased levels of NLR, SII and APRI maybe played the same roles as IL-6. Through the analysis, we found that only NLR and SII, but not other inflammatory indices, such as IL-6 and APRI, were significantly higher on admission in severe/critical pneumonia group than moderate pneumonia group (Fig. 1A-D). These results suggested that NLR and SII played a critical role in evaluating the severity of COVID-19.

\section{Characteristics of pregnant women with COVID-19 and newborns}

Of these 31 pregnant patients with COVID-19, five (16.1\%) were asymptomatic and one $(3 \cdot 2 \%)$ had an initial symptom of diarrhea. All the patients underwent fetal ultrasound examinations and no obvious abnormalities were found. These 31 pregnant patients were in the different trimesters, four (12.9\%) in first trimester, five $(16.1 \%)$ in second trimester and $22(71 \%)$ in third trimester (Table 3). Among the four patients in first trimester, three underwent uterine curettage by their own choice and one voluntarily chose fetal monitoring which was also chosen by five patients in second trimester and five patients in third trimester. Among the other third trimester patients, 13 chose cesarean section voluntarily, and four gave vaginal delivery. Finally, 17 livebirths were recorded. We collected breastmilk $(n=14)$, amniotic fluid $(n=2)$, placenta $(n=2)$, neonatal throat swab $(n=17)$ and neonatal anal swab $(n=5)$ to test the presence of SARS-CoV-2 by RT-PCR. SARS-CoV-2 was not detected in all these samples.

Additionally, five of these 17 neonates were premature but all were beyond 35 gestational weeks (Table 4). One neonate at 35 gestational weeks had a low birthweight of 2450g, with a 1-min Apgar score of seven and a 5-min Apgar score of eight. Other neonates had a one-min Apgar score of eight and a 5-min Apgar score of nine. All these newborns were in a stable condition. No fetal death, neonatal death or neonatal asphyxia were observed.

\section{Discussion}

Coronaviruses such as SARS-CoV and MERS- CoV, can cause severe adverse pregnancy outcomes, including miscarriage, intrauterine growth restriction, premature delivery, neonatal asphyxia and maternal death. ${ }^{15,24-26}$ However, the effects of SARS-CoV-2 infection on pregnancy is unclear, and so far there is no evidence of severity for mothers and newborns. Therefore, a cohort study is necessary and urgent to provide a reliable guideline for management of pregnant women with SARS-CoV-2 infection. To this end, we reported a cohort analysis of clinical outcomes in 31 pregnant women and 35 non-pregnant women with laboratory confirmed COVID-19. To our knowledge, this is the first cohort study that has been reported to date. Previously, a descriptive study demonstrated that the clinical characteristics of pregnant patients with COVID-19 resembled those of non-pregnant patients with COVID-19. ${ }^{12}$ However, we found a shorter interval from onset to hospitalization and severer COVID-19 in pregnant patients than non-pregnant patients with SARS-CoV-2 infection. This phenomenon could be partly explained by the special states of immune suppression and physiological adaptive changes during pregnancy. Infectious pneumonia is an essential cause of morbidity and mortality in pregnant women. For example, H1N1 virus caused a higher rate of hospital admission and an increasing risk for pregnancy complications in pregnant women than the general population. ${ }^{27}$ In our study, despite no death in pregnant women and neonates, our finding suggested that more attention should be paid to the pregnant women especially to those who had a history of exposure to the 
confirmed cases.

Additionally, pregnant women had insidious and atypical symptoms which might increase the risk of misdiagnosis. Fever was the most common symptom in the general population, as previously reported (over $80 \%)^{28,29}$ In our study, we found the similar pattern of symptoms in pregnant women, while the proportion of fever in pregnant women was far lower than that in non-pregnant women $(54.8 \%$ vs. $87.5 \%, p=0.006)$. Temperature screening has been the most proposed and useful method to identify the suspected patients in population. Thus, a low proportion of fever might increase the risk of missing the suspected patients in pregnant women. Furthermore, five (16.1\%) pregnant women who were diagnosed by laboratory confirmation of SARS-CoV-2 did not have any initial symptoms but had the history of exposure, and one (3.2\%) pregnant woman had an initial symptom of diarrhea. Importantly, chest CT scan of all patients showed moderate to severe inflammation in lungs. These findings plainly indicated that it was not enough to screen pregnant women for COVID-19 based solely on clinical symptoms. Monitoring, early detection and diagnosis are urgent and necessary for pregnant women, especially for those with a history of exposure.

There were numerous differences in the laboratory findings between pregnant patients and non-pregnant patients. Compared with nonpregnant patients, pregnant patients had more white blood cell and neutrophil counts, shorter prothrombin time and activated partial thromboplastin time, higher level of D-dimer, procalcitonin and aspartate aminotransferase. Moreover, there was no statistical difference in the proportion of abnormal levels of alanine transaminase and serum creatinine between these two groups, while pregnant patients had higher level of alanine transaminase and lower level of serum creatinine than non-pregnant patients. These abnormalities suggested that pregnant patients might have diminished cell-mediated immunity, hypercoagulable state, and increased hepatic injury compared with non-pregnant patients. These abnormalities were not unique between pregnant and non-pregnant patients with COVID-19. Previous studies showed that pregnant patients were more likely to have complications and adverse outcomes such as sepsis and hepatic injury with SARS-CoV and MERS-CoV infection. ${ }^{30,31}$

Monitoring the severity of COVID-19 is imperative for the adjustment of treatment strategies. Though analyzing cytokines in peripheral blood of 123 hospitalized patients with COVID-19, Wan et al. found that IL-6 were higher in severe patients than in mild patients. ${ }^{23}$ Another team observed the similar phenomenon and further demonstrated significant decrease of IL-6 after treatment for patients with severe COVID-19. ${ }^{22}$ Our study showed that pregnant patients had increased IL-6 level compared with non-pregnant patients, which is consistent with our previous finding that more pregnant patients were diagnosed as severe or critical pneumonia than non-pregnant patients. However, in our study, although the median level of IL-6 was higher in severe/critical group than moderate group, no significant difference was identified between these two groups. NLR and SII are the inflammatory markers which have been reported to be associated with the progress of disease. ${ }^{32,33} \mathrm{We}$ found that NLR and SII were positively correlated with the severity of COVID-19. It seems that these two inflammatory indices have a higher sensitivity than IL-6 on evaluating the severity of COVID-19. Besides, unlike IL-6, NLR and SII could be easily calculated from blood standard examination. A large, prospective study is necessary to investigate the value of NLR and SII in monitoring the severity of COVID-19.

Another focus of this study was to evaluate the possibility of vertical transmission of COVID-19. We did not find evidence of SARS-CoV-2 by RT-PCR in breastmilk, amniotic fluid, placenta, neonatal throat swab, and neonatal anal swab. These findings suggested that SARS-CoV-2 could not penetrate the placental and blood-milk barriers. This observation confirms 5 premature delivery and 12 full-term 
birth including 17 pregnant patients and 17 neonates, showing that COVID-19 was negative in all the neonates. Moreover, we reviewed the previous reports about pregnant women with SARS-CoV and MERS-CoV infection. There was no evidence that SARS-CoV and MERS-CoV could be transmitted from infected mothers to infants during pregnancy.

This study has several limitations. First, we only included the hospitalized patients with laboratory confirmed COVID-19. It could be more methodologically sound to include both pregnant outpatients and inpatients in the analysis to obtain a lees biased estimate comprehensive understanding of the effect of COVID-19 on pregnancy. Second, patient's information such as pregnant outcomes in first and second trimester were incomplete and unavailable at the time of analysis. Third, selection bias might be introduced using 35 non-pregnant patients of reproductive age as control cohort. To clarify, we made a baseline comparison between these 35 non-pregnant patients and all female patients of reproductive age from Hunan province near Wuhan where COVID-19 broke out and found no difference (Table S2). Finally, considering the limited number in this study, we should treat the results with more caution. Non-significant $\mathrm{P}$ value still could not rule out the possibility of false negative results when comparing pregnant and non-pregnant patients.

In summary, pregnant women with COVID-19 had insidious and atypical symptoms which increased the risk of misdiagnosis. SII and NLR could be useful markers to evaluate the severity of the COVID-19. There was no evidence of vertical transmission during pregnancy. These findings will be crucial to inform prophylactic and therapeutic strategies for pregnant women with COVID-19.

\section{Contributors}

MY and XC designed the study. LZ, CF, SY and HC collected the data. MY and MS were involved in data cleaning, and verification. MY, MS, and GD analyzed the data. GD and MY drafted the manuscript. MY, GD and MS contributed to the interpretation of the results and critical revision of the manuscript for important intellectual content and approved the final version of the manuscript. All authors have read and approved the final manuscript. MY, XC SY and HC are the study guarantors. DB independently reviewed all data and analysis due to my expertise in emerging infectious disease.

\section{Declaration of interests}

All authors declare no competing interests.

\section{Data sharing}

No additional data are available.

\section{Acknowledgements}

We thank all the hospital staff members for their efforts in collecting the information that used in this study; thank the patients who participated in this study, their families, and the medical, nursing, and research staff at the study centers. 


\section{Reference}

1. Chinazzi M, Davis JT, Ajelli M, et al. The effect of travel restrictions on the spread of the 2019 novel coronavirus (COVID-19) outbreak. Science 2020.

2. Guan WJ, Ni ZY, Hu Y, et al. Clinical Characteristics of Coronavirus Disease 2019 in China. $N$ Engl J Med 2020.

3. Huang C, Wang Y, Li X, et al. Clinical features of patients infected with 2019 novel coronavirus in Wuhan, China. Lancet 2020; 395(10223): 497-506.

4. Ng OT, Marimuthu K, Chia PY, et al. SARS-CoV-2 Infection among Travelers Returning from Wuhan, China. N Engl J Med 2020.

5. Zhu N, Zhang D, Wang W, et al. A Novel Coronavirus from Patients with Pneumonia in China, 2019. N Engl J Med 2020; 382(8): 727-33.

6. WHO. Coronavirus disease (COVID-2019) situation reports. https://www.who.int/emergencies/diseases/novel-coronavirus-2019/situation-reports/.

7. Wu JT, Leung K, Leung GM. Nowcasting and forecasting the potential domestic and international spread of the 2019-nCoV outbreak originating in Wuhan, China: a modelling study. Lancet 2020; 395(10225): 689-97.

8. Wu Z, McGoogan JM. Characteristics of and Important Lessons From the Coronavirus Disease 2019 (COVID-19) Outbreak in China: Summary of a Report of 72314 Cases From the Chinese Center for Disease Control and Prevention. JAMA 2020.

9. Organization WH. Coronavirus disease (COVID-19) outbreak (http://www.who.int).

10. Qiao J. What are the risks of COVID-19 infection in pregnant women? Lancet 2020; 395(10226): 760-2.

11. Stower H. Lack of maternal-fetal SARS-CoV-2 transmission. Nat Med 2020; 26(3): 312.

12. Chen $\mathrm{H}$, Guo J, Wang $\mathrm{C}$, et al. Clinical characteristics and intrauterine vertical transmission potential of COVID-19 infection in nine pregnant women: a retrospective review of medical records. Lancet 2020; 395(10226): 809-15.

13. Zhu H, Wang L, Fang C, et al. Clinical analysis of 10 neonates born to mothers with 2019-nCoV pneumonia. Transl Pediatr 2020; 9(1): 51-60.

14. Wang X, Zhou Z, Zhang J, et al. A case of 2019 Novel Coronavirus in a pregnant woman with preterm delivery. Clin Infect Dis 2020.

15. Favre G, Pomar L, Musso D, et al. 2019-nCoV epidemic: what about pregnancies? Lancet 2020; 395(10224): e40.

16. The notice of launching guideline on diagnosis and treatment of the novel coronavirus pneumonia (6th edition) (in Chinese) http://www.gov.cn/zhengce/zhengceku/2020-02/19/content_5480948.htm.

17. WHO. Clinical management of severe acute respiratory infection when novel coronavirus (nCoV) infection

is

suspected.

https://www.who.int/docs/default-source/coronaviruse/clinical-management-of-novel-cov.pdf.

18. Channappanavar R, Perlman S. Pathogenic human coronavirus infections: causes and consequences of cytokine storm and immunopathology. Semin Immunopathol 2017; 39(5): 529-39.

19. Huang KJ, Su IJ, Theron M, et al. An interferon-gamma-related cytokine storm in SARS patients. J Med Virol 2005; 75(2): 185-94.

20. Jiang $\mathrm{Y}, \mathrm{Xu}$ J, Zhou $\mathrm{C}$, et al. Characterization of cytokine/chemokine profiles of severe acute respiratory syndrome. Am J Respir Crit Care Med 2005; 171(8): 850-7.

21. Ying T, Prabakaran P, Du L, et al. Junctional and allele-specific residues are critical for 
MERS-CoV neutralization by an exceptionally potent germline-like antibody. Nat Commun 2015; 6 : 8223.

22. Liu T, Zhang J, Yang Y, et al. The potential role of IL-6 in monitoring severe case of coronavirus disease 2019. medRxiv 2020: 2020.03.01.20029769.

23. Wan S, Yi Q, Fan S, et al. Characteristics of lymphocyte subsets and cytokines in peripheral blood of 123 hospitalized patients with 2019 novel coronavirus pneumonia (NCP). medRxiv 2020: 2020.02.10.20021832.

24. Alfaraj SH, Al-Tawfiq JA, Memish ZA. Middle East Respiratory Syndrome Coronavirus (MERS-CoV) infection during pregnancy: Report of two cases \& review of the literature. J Microbiol Immunol Infect 2019; 52(3): 501-3.

25. Lam CM, Wong SF, Leung TN, et al. A case-controlled study comparing clinical course and outcomes of pregnant and non-pregnant women with severe acute respiratory syndrome. BJOG 2004; 111(8): 771-4.

26. Wong SF, Chow KM, Leung TN, et al. Pregnancy and perinatal outcomes of women with severe acute respiratory syndrome. Am J Obstet Gynecol 2004; 191(1): 292-7.

27. Jamieson DJ, Honein MA, Rasmussen SA, et al. H1N1 2009 influenza virus infection during pregnancy in the USA. Lancet 2009; 374(9688): 451-8.

28. Chen N, Zhou M, Dong X, et al. Epidemiological and clinical characteristics of 99 cases of 2019 novel coronavirus pneumonia in Wuhan, China: a descriptive study. Lancet 2020; 395(10223): 507-13.

29. Wang D, Hu B, Hu C, et al. Clinical Characteristics of 138 Hospitalized Patients With 2019 Novel Coronavirus-Infected Pneumonia in Wuhan, China. JAMA 2020.

30. Rasmussen SA, Smulian JC, Lednicky JA, et al. Coronavirus Disease 2019 (COVID-19) and Pregnancy: What obstetricians need to know. Am J Obstet Gynecol 2020.

31. Schwartz DA, Graham AL. Potential Maternal and Infant Outcomes from (Wuhan) Coronavirus 2019-nCoV Infecting Pregnant Women: Lessons from SARS, MERS, and Other Human Coronavirus Infections. Viruses 2020; 12(2).

32. Deng G, Yao L, Zeng F, et al. Nomogram For Preoperative Prediction Of Microvascular Invasion Risk In Hepatocellular Carcinoma. Cancer Manag Res 2019; 11: 9037-45.

33. Jomrich G, Paireder M, Kristo I, et al. High Systemic Immune-Inflammation Index is an Adverse Prognostic Factor for Patients With Gastroesophageal Adenocarcinoma. Ann Surg 2019. 
medRxiv preprint doi: https://doi.org/10.1101/2020.04.07.20053744; this version posted April 11, 2020. The copyright holder for this preprint (which was not certified by peer review) is the author/funder, who has granted medRxiv a license to display the preprint in perpetuity.

All rights reserved. No reuse allowed without permission.

Table 1. Clinical characteristics of pregnant vs. nonpregnant women of childbearing age infected with COVID-19.

\begin{tabular}{|c|c|c|c|c|}
\hline Characteristics & Total $(\mathrm{N}=66)$ & $\begin{array}{l}\text { Pregnant } \\
(n=31)\end{array}$ & $\begin{array}{l}\text { Nonpregnant } \\
(\mathbf{n}=35)\end{array}$ & $P$ \\
\hline \multicolumn{5}{|l|}{ Symptoms, n (\%) } \\
\hline Fever & $47(71 \cdot 2)$ & $17(54 \cdot 8)$ & $30(85 \cdot 7)$ & 0.006 \\
\hline Dry cough & $31(47 \cdot 0)$ & $15(48 \cdot 4)$ & $16(45 \cdot 7)$ & $0 \cdot 828$ \\
\hline Fatigue & $18(27 \cdot 3)$ & $6(19 \cdot 4)$ & $12(34 \cdot 3)$ & $0 \cdot 174$ \\
\hline Dyspnea & $17(25 \cdot 8)$ & $8(25 \cdot 8)$ & $9(25 \cdot 7)$ & 0.993 \\
\hline Expectoration & $15(22 \cdot 7)$ & $5(16 \cdot 1)$ & $10(28 \cdot 6)$ & $0 \cdot 229$ \\
\hline Diarrhea & $10(15 \cdot 2)$ & $2(6 \cdot 5)$ & $8(22.9)$ & 0.064 \\
\hline Myalgia & $9(13 \cdot 6)$ & $3(9 \cdot 7)$ & $6(17 \cdot 1)$ & $0 \cdot 378$ \\
\hline Anorexia & $7(10 \cdot 6)$ & $3(9 \cdot 7)$ & $4(11.4)$ & $0 \cdot 818$ \\
\hline Nausea & $7(10 \cdot 6)$ & $3(9 \cdot 7)$ & $4(11 \cdot 4)$ & $0 \cdot 818$ \\
\hline Dizziness & $4(6 \cdot 1)$ & $2(6 \cdot 5)$ & $2(5 \cdot 7)$ & $0 \cdot 900$ \\
\hline Vomiting & $4(6 \cdot 1)$ & $2(6 \cdot 5)$ & $2(5 \cdot 7)$ & 0.900 \\
\hline Pharyngalgia & $2(3 \cdot 0)$ & $1(3 \cdot 2)$ & $1(2 \cdot 9)$ & $0.723^{*}$ \\
\hline Headache & $1(1 \cdot 5)$ & $0(0 \cdot 0)$ & $1(2 \cdot 9)$ & $0.530^{*}$ \\
\hline Abdominal pain & $1(1 \cdot 5)$ & $1(3 \cdot 2)$ & $0(0 \cdot 0)$ & $0.284^{*}$ \\
\hline \multicolumn{5}{|l|}{ Severity, n (\%) } \\
\hline Moderate & $52(78 \cdot 8)$ & $21(67 \cdot 7)$ & $31(88 \cdot 6)$ & $0 \cdot 039$ \\
\hline Severe / critical & $14(21 \cdot 2)$ & $10(32 \cdot 3)$ & $4(11 \cdot 4)$ & \\
\hline Onset to hospitalization (days), mean $\pm \mathrm{SD}$ & $10 \cdot 7 \pm 8 \cdot 1$ & $7 \cdot 8 \pm 7 \cdot 0$ & $13 \cdot 2 \pm 8 \cdot 2$ & $0 \cdot 005$ \\
\hline Discharged patients, n (\%) & $52(78 \cdot 8)$ & $22(71 \cdot 0)$ & $30(85 \cdot 7)$ & $0 \cdot 144$ \\
\hline Hospital stay (day), mean \pm SD & $17 \cdot 8 \pm 6 \cdot 1$ & $17 \cdot 3 \pm 6 \cdot 9$ & $18 \cdot 2 \pm 5 \cdot 6$ & $0 \cdot 595$ \\
\hline $\mathrm{MAP}(\mathrm{mmHg})$, mean $\pm \mathrm{SD}$ & $92 \cdot 2 \pm 10 \cdot 1$ & $87 \cdot 2 \pm 10 \cdot 4$ & $96 \cdot 7 \pm 7 \cdot 6$ & $<0 \cdot 001$ \\
\hline Heart rate (/min), mean \pm SD & $90 \cdot 3 \pm 15 \cdot 0$ & $93 \cdot 0 \pm 15 \cdot 9$ & $88 \cdot 0 \pm 13 \cdot 9$ & $0 \cdot 176$ \\
\hline Respiratory rate (/min), mean \pm SD & $20 \cdot 7 \pm 3 \cdot 0$ & $20 \cdot 7 \pm 2 \cdot 6$ & $20 \cdot 6 \pm 3 \cdot 4$ & 0.849 \\
\hline
\end{tabular}

SD: standard deviation; MAP: mean arterial pressure.

* Fisher's exact probability. 
Table 2. Laboratory and radiological findings in pregnant vs. nonpregnant women of childbearing age infected with COVID-19.

\begin{tabular}{|c|c|c|c|c|}
\hline Laboratory indicators & Total $(\mathrm{N}=66)$ & Pregnant (n=31) & Nonpregnant $(\mathbf{n}=35)$ & $P$ \\
\hline $\mathrm{WBC}\left(\times 10^{9} / \mathrm{L}\right)$, mean $\pm \mathrm{SD}$ & $5 \cdot 97 \pm 2 \cdot 70$ & $6 \cdot 95 \pm 2 \cdot 98$ & $5 \cdot 11 \pm 2 \cdot 13$ & 0.005 \\
\hline$<4 \times 10^{9} / \mathrm{L}, \mathrm{n}(\%)$ & $17(25 \cdot 8)$ & $4(12 \cdot 9)$ & $13(37 \cdot 1)$ & 0.027 \\
\hline $4-10 \times 10^{9} / \mathrm{L}, \mathrm{n}(\%)$ & $43(65 \cdot 2)$ & $22(71 \cdot 0)$ & $21(60 \cdot 9)$ & \\
\hline$>10 \times 10^{9} / \mathrm{L}, \mathrm{n}(\%)$ & $6(9 \cdot 1)$ & $5(16 \cdot 1)$ & $1(2 \cdot 9)$ & \\
\hline Neutrophil count $\left(\times 10^{9} / \mathrm{L}\right)$, mean $\pm \mathrm{SD}$ & $4 \cdot 19 \pm 2 \cdot 46$ & $5 \cdot 28 \pm 2 \cdot 59$ & $3 \cdot 21 \pm 1 \cdot 89$ & $<0 \cdot 001$ \\
\hline$<1.8 \times 10^{9} / \mathrm{L}, \mathrm{n}(\%)$ & $7(10 \cdot 6)$ & $0(0)$ & $7(20 \cdot 0)$ & $0 \cdot 017$ \\
\hline $1.8-6.3 \times 10^{9} / \mathrm{L}, \mathrm{n}(\%)$ & $47(71 \cdot 2)$ & $21(67 \cdot 7)$ & $26(74 \cdot 3)$ & \\
\hline$>6.3 \times 10^{9} / \mathrm{L}, \mathrm{n}(\%)$ & $12(18 \cdot 2)$ & $10(32 \cdot 3)$ & $2(5 \cdot 7)$ & \\
\hline Lymphocyte count $\left(\times 10^{9} / \mathrm{L}\right)$, mean $\pm \mathrm{SD}$ & $1 \cdot 32 \pm 0 \cdot 54$ & $1 \cdot 24 \pm 0 \cdot 57$ & $1 \cdot 40 \pm 0 \cdot 50$ & $0 \cdot 243$ \\
\hline Monocyte count, mean \pm SD & $0 \cdot 38 \pm 0 \cdot 16$ & $0 \cdot 36 \pm 0 \cdot 18$ & $0 \cdot 40 \pm 0 \cdot 14$ & 0.331 \\
\hline Platelet count $\left(\times 10^{9} / \mathrm{L}\right)$, mean $\pm \mathrm{SD}$ & $218 \cdot 9 \pm 66 \cdot 6$ & $206 \cdot 0 \pm 57 \cdot 8$ & $230 \cdot 3 \pm 72 \cdot 4$ & $0 \cdot 141$ \\
\hline $\mathrm{PT}(\mathrm{sec})$, mean $\pm \mathrm{SD}^{\#}$ & $12 \cdot 8 \pm 1 \cdot 4$ & $11 \cdot 9 \pm 1 \cdot 5$ & $13 \cdot 6 \pm 0 \cdot 7$ & $<0 \cdot 001$ \\
\hline$<11 \mathrm{sec}, \mathrm{n}(\%)$ & $1(1 \cdot 6)$ & $1(3 \cdot 3)$ & $0(0 \cdot 0)$ & $<0 \cdot 001$ \\
\hline $11-13 \mathrm{sec}, \mathrm{n}(\%)$ & $32(50 \cdot 0)$ & $26(86 \cdot 7)$ & $6(17 \cdot 6)$ & \\
\hline$>13 \mathrm{sec}, \mathrm{n}(\%)$ & $31(48 \cdot 4)$ & $3(10 \cdot 0)$ & $28(82 \cdot 4)$ & \\
\hline APTT (sec), mean $\pm \mathrm{SD}^{\#}$ & $37 \cdot 9 \pm 4 \cdot 9$ & $36 \cdot 1 \pm 4 \cdot 1$ & $39 \cdot 6 \pm 5 \cdot 1$ & 0.005 \\
\hline D-dimer $(\mathrm{mg} / \mathrm{L})$, median $(\mathrm{IQR})^{\#}$ & $0 \cdot 60(0 \cdot 31-1 \cdot 02)$ & $0.91(0 \cdot 58-1 \cdot 32)$ & $0 \cdot 36(0 \cdot 26-0 \cdot 71)$ & $0.003^{*}$ \\
\hline$>0.5 \mathrm{mg} / \mathrm{L}, \mathrm{n}(\%)^{\#}$ & $31(47 \cdot 7)$ & $25(80 \cdot 6)$ & $12(35 \cdot 3)$ & $<0 \cdot 001$ \\
\hline Procalcitonin (ng/ml), median (IQR) ${ }^{\#}$ & $0.05(0.03-0 \cdot 08)$ & $0.08(0 \cdot 05-0 \cdot 32)$ & $0 \cdot 04(0 \cdot 02-0 \cdot 04)$ & $<0 \cdot 001^{*}$ \\
\hline$>0.1 \mathrm{ng} / \mathrm{ml}, \mathrm{n}(\%)^{\#}$ & $51(81 \cdot 0)$ & $30(96 \cdot 8)$ & $21(65 \cdot 6)$ & 0.002 \\
\hline AST (U/L), median (IQR) & $19 \cdot 0(16 \cdot 0-31 \cdot 0)$ & $23 \cdot 0(18 \cdot 0-40 \cdot 0)$ & $18 \cdot 0(15 \cdot 0-22 \cdot 0)$ & $0 \cdot 010^{*}$ \\
\hline$>40 \mathrm{U} / \mathrm{L}, \mathrm{n}(\%)$ & $9(13 \cdot 6)$ & $7(22 \cdot 6)$ & $2(5 \cdot 7)$ & 0.046 \\
\hline $\operatorname{ALT}(\mathrm{U} / \mathrm{L})$, median (IQR) & $16 \cdot 0(10 \cdot 0-31 \cdot 0)$ & $24 \cdot 0(10 \cdot 0-35 \cdot 0)$ & $11 \cdot 0(8 \cdot 0-24 \cdot 0)$ & $0.032^{*}$ \\
\hline$>40 \mathrm{U} / \mathrm{L}, \mathrm{n}(\%)$ & $10(15 \cdot 2)$ & $6(19 \cdot 4)$ & $4(11 \cdot 4)$ & $0 \cdot 370$ \\
\hline
\end{tabular}




\begin{tabular}{|c|c|c|c|c|}
\hline Serum creatinine $(\mu \mathrm{mol} / \mathrm{L})$, mean $\pm \mathrm{SD}$ & $49 \cdot 9 \pm 8 \cdot 5$ & $46 \cdot 5 \pm 7 \cdot 0$ & $52 \cdot 9 \pm 8 \cdot 7$ & 0.002 \\
\hline $\mathrm{BUN}(\mathrm{mmol} / \mathrm{L})$, mean $\pm \mathrm{SD}$ & $2 \cdot 91 \pm 0 \cdot 90$ & $2 \cdot 92 \pm 0 \cdot 96$ & $2 \cdot 89 \pm 0 \cdot 87$ & $0 \cdot 896$ \\
\hline $\mathrm{LDH}(\mathrm{U} / \mathrm{L})$, mean $\pm \mathrm{SD}$ & $208 \cdot 0 \pm 58 \cdot 2$ & $199 \cdot 7 \pm 54 \cdot 7$ & $215 \cdot 3 \pm 61 \cdot 0$ & $0 \cdot 280$ \\
\hline $\mathrm{CRP}(\mathrm{mg} / \mathrm{L})$, median $(\mathrm{IQR})^{\#}$ & $3 \cdot 9(1 \cdot 1-23 \cdot 1)$ & $6 \cdot 6(1 \cdot 4-32 \cdot 2)$ & $3 \cdot 4(0 \cdot 5-11 \cdot 6)$ & $0 \cdot 338^{*}$ \\
\hline IL-6 (pg/ml), median (IQR) ${ }^{\#}$ & $2 \cdot 90(1.06-4 \cdot 75)$ & $3 \cdot 7(2 \cdot 6-5 \cdot 6)$ & $1 \cdot 1(1 \cdot 1-3 \cdot 1)$ & $<0.001^{*}$ \\
\hline NLR, median (IQR) & $2.58(1.85-4.73)$ & $4 \cdot 21(2 \cdot 88-5 \cdot 61)$ & $2 \cdot 31(1 \cdot 33-2 \cdot 54)$ & $<0.001^{*}$ \\
\hline LMR, median (IQR) & $3 \cdot 58(2 \cdot 75-4 \cdot 71)$ & $3 \cdot 55(2 \cdot 74-5 \cdot 00)$ & $3 \cdot 59(2 \cdot 76-4 \cdot 37)$ & $0 \cdot 787^{*}$ \\
\hline PLR, median (IQR) & $160 \cdot 3(135 \cdot 6-226 \cdot 4)$ & $156 \cdot 8(144 \cdot 4-246 \cdot 2)$ & $170 \cdot 9(122 \cdot 0-211 \cdot 0)$ & $0 \cdot 612^{*}$ \\
\hline SII, median (IQR) & $567 \cdot 6(376 \cdot 2-968 \cdot 0)$ & $841 \cdot 1(543 \cdot 2-1175 \cdot 4)$ & $464 \cdot 9(235 \cdot 7-761 \cdot 0)$ & $0 \cdot 001^{*}$ \\
\hline ANRI, median (IQR) & $6 \cdot 25(3 \cdot 53-10 \cdot 29)$ & $4 \cdot 30(3 \cdot 01-10 \cdot 03)$ & $6 \cdot 96(4 \cdot 83-10 \cdot 47)$ & $0 \cdot 110^{*}$ \\
\hline APRI, median (IQR) & $0 \cdot 24(0 \cdot 18-0 \cdot 37)$ & $0 \cdot 33(0 \cdot 24-0 \cdot 42)$ & $0 \cdot 21(0 \cdot 16-0 \cdot 29)$ & $0 \cdot 001^{*}$ \\
\hline Distribution of patchy shadows & $64(97 \cdot 0)$ & $30(96 \cdot 8)$ & $34(97 \cdot 1)$ & 0.527 \\
\hline or ground glass opacity, $\mathrm{n}(\%)$ & & & & \\
\hline
\end{tabular}

SD: standard deviation; IQR: interquartile range; WBC: white blood cell; PT: prothrombin time; APTT: activated partial thromboplastin time; ALT: Alanine transaminase;

AST: aspartate aminotransferase; BUN: blood urea nitrogen; LDH: lactic dehydrogenase; CRP: C-reactive protein; IL: interleukin; NLR: neutrophil-to-lymphocyte ratio;

LMR: lymphocyte-to-monocyte ratio; PLR: platelet-to-lymphocyte ratio; SII: systemic Immune-inflammation Index; ANRI: AST-to-neutrophil ratio index; APRI:

AST-to-platelet ratio index.

* Tested by Wilcoxon rank sum test.

${ }^{\#}$ Missing data: PT (n=2), APTT (n=3), D-dimer ( $\left.=1\right)$, procalcitonin ( $\left.\mathrm{n}=3\right), \operatorname{CRP}(\mathrm{n}=1), \mathrm{IL}-6(\mathrm{n}=3)$. 
Table 3. Comparison of characteristics of pregnant women with COVID-19 and newborn.

\begin{tabular}{|c|c|c|c|c|}
\hline \multirow[b]{2}{*}{ Characteristics } & \multirow[b]{2}{*}{ Pregnant women $(\mathrm{N}=31)$} & \multicolumn{3}{|c|}{ Gestational age (weeks) } \\
\hline & & $0-12(n=4)$ & $13-27(n=5)$ & $\geq 28(n=22)$ \\
\hline Fetal heart rate, mean \pm SD & $143 \cdot 1 \pm 14 \cdot 1$ & N/A & $145 \cdot 6 \pm 8 \cdot 0$ & $142 \cdot 6 \pm 15 \cdot 6$ \\
\hline Abnormal ultrasound, n, (\%) & $0(0 \cdot 0)$ & $0(0 \cdot 0)$ & $0(0 \cdot 0)$ & $0(0 \cdot 0)$ \\
\hline \multicolumn{5}{|l|}{ Obstetric management, n (\%) } \\
\hline Fetal monitoring & $11(35 \cdot 5)$ & $1(25 \cdot 0)$ & $5(100 \cdot 0)$ & $5(22.7)$ \\
\hline Curettage & $3(9 \cdot 6)$ & $3(75 \cdot 0)$ & $0(0 \cdot 0)$ & $0(0 \cdot 0)$ \\
\hline Cesarean & $13(41 \cdot 9)$ & $0(0 \cdot 0)$ & $0(0 \cdot 0)$ & $13(59 \cdot 1)$ \\
\hline Eutocia & $4(12 \cdot 9)$ & $0(0 \cdot 0)$ & $0(0 \cdot 0)$ & $4(18 \cdot 2)$ \\
\hline \multicolumn{5}{|l|}{ RT-PCR assay for SARS-CoV-2, n (\%) } \\
\hline Maternal throat swab $(\mathrm{n}=17)$ & $17(100 \cdot 0)$ & N/A & N/A & N/A \\
\hline Breastmilk $(\mathrm{n}=14)$ & $0(0 \cdot 0)$ & N/A & N/A & N/A \\
\hline Amniotic fluid ( $\mathrm{n}=2$ ) & $0(0 \cdot 0)$ & N/A & N/A & N/A \\
\hline Placenta $(\mathrm{n}=2)$ & $0(0 \cdot 0)$ & N/A & N/A & N/A \\
\hline Neonatal throat swab $(n=17)$ & N/A & N/A & N/A & $0(0 \cdot 0)$ \\
\hline Neonatal anal swab $(n=5)$ & N/A & N/A & N/A & $0(0 \cdot 0)$ \\
\hline
\end{tabular}

N/A: not available or not determined. 
medRxiv preprint doi: https://doi.org/10.1101/2020.04.07.20053744; this version posted April 11, 2020. The copyright holder for this preprint (which was not certified by peer review) is the author/funder, who has granted medRxiv a license to display the preprint in perpetuity.

All rights reserved. No reuse allowed without permission.

Table 4. Neonatal outcome.

\begin{tabular}{lll}
\hline & \multicolumn{2}{c}{ Parturient puerpera (N=17) } \\
\cline { 2 - 3 } Characteristics & Premature delivery (n=5) & Full-term birth (n=12) \\
\hline Gestational age at delivery (weeks), median (range) & $37(35-37)$ & $39(38-41)$ \\
Birthweight (g), median (range) & $2580(2450-3130)$ & $3035(2830-4100)$ \\
Low birthweight, n $(\%)(<2500 \mathrm{~g})$ & $1(20 \cdot 0)$ & $0(0 \cdot 0)$ \\
Apgar score & & \\
$\quad 1$ min, median (range) & $8(7-8)$ & 8 \\
$\quad 9$ min, median (range) & $9(8-9)$ & 9 \\
Severe neonatal asphyxia & $0(0 \cdot 0)$ & $0(0 \cdot 0)$ \\
Neonatal death & $0(0 \cdot 0)$ & $0(0 \cdot 0)$ \\
Fetal death or stillbirth & $0(0 \cdot 0)$ & $0(0 \cdot 0)$ \\
\hline
\end{tabular}




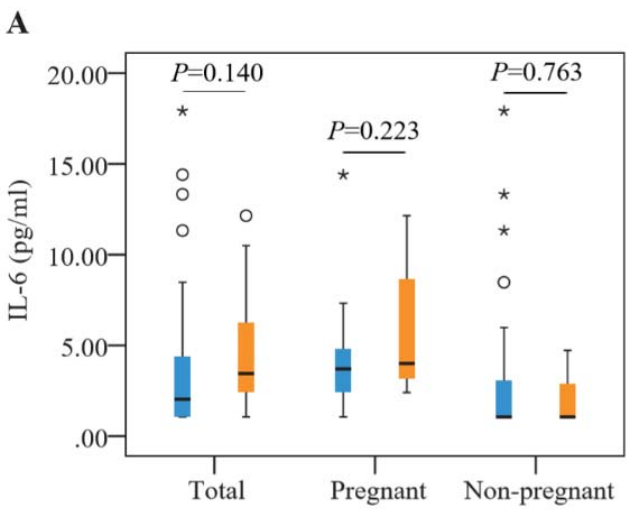

B

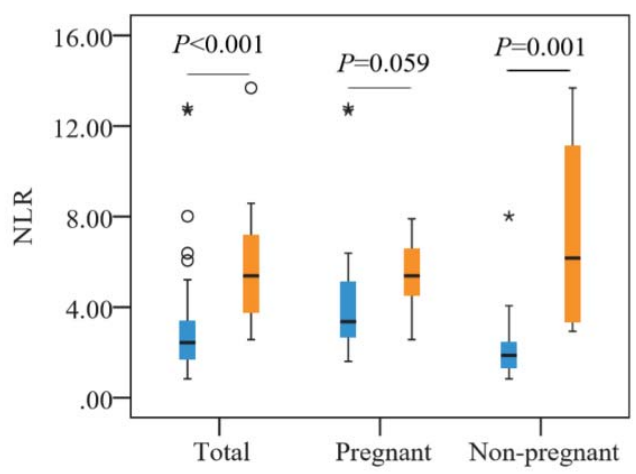

C

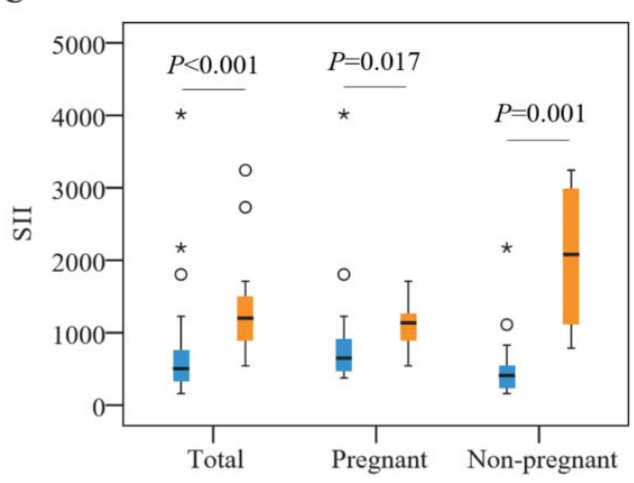

D

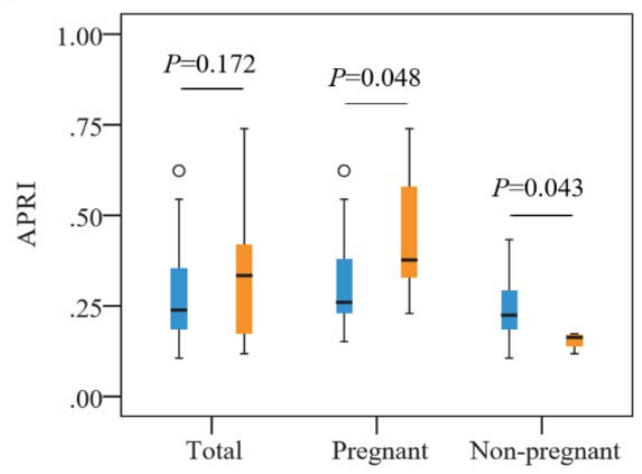

Figure 1. Correlation between inflammatory indices and severity of COVID-19.

Interleukin (IL)-6 (A), systematic immune-inflammation-based prognostic index (SII) (B), neutrophil-to-lymphocyte ratio (NLR) (C), aspartate aminotransferase-to-platelet ratio index (APRI) (D) levels in general population, pregnant and non-pregnant women between moderate and severe/critical COVID-19. 
medRxiv preprint doi: https://doi.org/10.1101/2020.04.07.20053744; this version posted April 11, 2020. The copyright holder for this preprint (which was not certified by peer review) is the author/funder, who has granted medRxiv a license to display the preprint in perpetuity.

All rights reserved. No reuse allowed without permission. 